\title{
Der Weltärztebund hat das Genfer Gelöbnis revidiert
}

\author{
Urban Wiesing ${ }^{a}$, Ramin Parsa-Parsi ${ }^{b}$ \\ a Prof. Dr. med. Dr. phil., Direktor des Instituts für Ethik und Geschichte der Medizin, Universität Tübingen; ${ }^{b}$ Dr. med., MPH, Leiter des Dezernats für \\ Internationale Angelegenheiten, Bundesärztekammer, Berlin; Mitglied im Vorstand des Weltärztebundes, Leiter der Arbeitsgruppe zur Revision des Genfer \\ Gelöbnisses
}

Auf seiner 68. Generalversammlung am 14. Oktober 2017 in Chicago hat die Generalversammlung des Weltärztebundes eine überarbeitete Version des Genfer Gelöbnisses verabschiedet, die einige bedeutende Änderungen und Ergänzungen aufweist. ${ }^{1}$ Damit wurde eines der wichtigsten Dokumente des Weltärztebundes revidiert, vergleichbar in seiner Bedeutung mit der Deklaration von Helsinki über ethische Grundsätze für die medizinische Forschung am Menschen. Das Genfer Gelöbnis kann auf eine Geschichte zurückblicken: Es wurde 1948 auf der 2. Generalversammlung des Weltärztebundes unter dem Eindruck des Zweiten Weltkrieges und der Gräueltaten unter Mitwirkung von Ärzten während der Nazi-Herrschaft verfasst. Das Gelöbnis sollte helfen, das Vertrauen der Patienten in die Ärzteschaft wiederherzustellen. Es wurde in den Jahren 1968 (Sydney), 1983 (Venedig) und 1994 (Stockholm) geringfügig revidiert sowie 2005 und 2006 an wenigen Stellen redaktionell überarbeitet. Es fasst die grundlegenden ethischen Prinzipien ärztlichen Handelns zusammen. Der französische Titel Serment d'Hippocrate, formule de Genève verweist auf die lange Tradition, in der sich das Gelöbnis sieht.

\section{Prinzipien der Revision}

Der Weltärztebund benannte die Ärzteverbände aus Indien, der Türkei, Israel, Schweden und den USA als Mitglieder der für die Revision zuständigen Arbeitsgruppe und beauftragte die deutsche Bundesärztekammer mit deren Leitung. Dem kommt in Anbetracht der Entstehungsgeschichte der Deklaration von Genf eine besondere Bedeutung zu. Die Zusammensetzung der Arbeitsgruppe schloss bewusst Mitglieder mit verschiedenem kulturellem, religiösem und ethnischem Hintergrund ein. Aufgrund der grossen Bedeutung des Gelöbnisses für den Weltärztebund wurde eine Bearbeitungszeit von 2 Jahren veranschlagt.

Die Arbeitsgruppe war sich schnell einig, dass die Revision Charakter, Umfang, Ausrichtung und Adressaten des Genfer Gelöbnisses belassen sollte. Zudem sollten nur Änderungen vorgenommen werden, für die es starke Argumente gäbe. Im Rahmen einer öffentlichen Konsultation hatten interessierte Experten und Stakeholder die Möglichkeit, mit Änderungsvorschlägen an der Revision teilzunehmen. Die Mitglieder des Weltärztebundes hatten zudem mehrfach die Gelegenheit, den überarbeiteten Entwurf zu kommentieren. Die Arbeitsgruppe prüfte alle Eingaben und wog deren Berücksichtigung anhand der genannten Prinzipien ab.

\section{Die Ergänzungen und Änderungen}

Die Revision hat in verschiedener Hinsicht bedeutende Ergänzungen und Änderungen vorgenommen. Als wichtigste Ergänzung darf dabei der Satz gelten, dass Ärzte die Autonomie ihrer Patienten zu respektieren haben. Wie auch der Hippokratisch Eid, so hatte das Genfer Gelöbnis bisher den Respekt der Selbstbestimmung eines Patienten nicht erwähnt. Damit stand es im Widerspruch zu zahlreichen anderen Dokumenten des Weltärztebundes, so der Deklaration von Helsinki, die für die klinische Forschung unmissverständlich und detailliert fordert, die Patientenautonomie zu respektieren. Mit dieser Änderung hat nun das zentrale ethische Gelöbnis der ärztlichen Profession die Selbstbestimmung der Patienten aufgenommen: «I WILL RESPECT the autonomy and dignity of my patient.» Die Revision hat zudem versucht, einige Formulierungen zeitgemässer zu gestalten. So wurde der im Englischen mit einer religiösen Konnotation besetzte Begriff 'consecrate' (weihen) durch 'dedicate' (widmen) ersetzt: «I SOLEMNLY PLEDGE to eonserate dedicate my life to the service of humanity.» Ebenso wurde die Verbindung zur Tradition des Berufsstandes nüchterner gefasst: «I WILL FOSTER MAINTAIN by allmeans in my power, the honour and noble traditions of the medical profession.» Der Satz «MY COLLEAGUES will be my sisters and brothers» wurde ebenfalls als sprachlich veraltet empfunden, deshalb gestrichen und der Inhalt durch die Ergänzung "colleagues and students» in den Satz aufgenommen: «I WILL GIVE to my teachers, colleagues, and students the respect and gratitude that is their due.» 
Die Erweiterung um Studenten beruht auch auf einer Intervention der Internationalen Vereinigung medizinischer Studentenorganisationen (International Federation of Medical Students' Associations - IFMSA). Deren Vertreter hatten den Weltärztebund darauf aufmerksam gemacht, dass in der bisherigen Fassung die Schüler zwar ihren Lehrern Respekt zu zollen haben, nicht aber umgekehrt. Die IFMSA hat in dieser Frage zu Recht Reziprozität eingefordert, die in dem 2500 Jahre alten Hippokratischen Eid bereits enthalten war. Durch den neu formulierten Satz ist dies nunmehr ausgedrückt.

Die neue Revision adressiert den Umgang mit Wissen. Nun schreibt das Genfer Gelöbnis vor, dass der Arzt das Wissen zu teilen habe, um den Patienten und der Gesundheitsversorgung zu dienen: «I WILL SHARE my medical knowledge for the benefit of the patient and the advancement of healthcare.»

Neu ist auch ein Satz, der sich auf den Arzt, seine Gesundheit und sein Befinden bezieht. Es ist aus zahlreichen Untersuchungen bekannt, dass übermüdete und überarbeitete Ärzte Gefahr laufen, schlechtere Medizin zu praktizieren. ${ }^{2}$ Das nimmt das Genfer Gelöbnis nun auf, indem es fordert, dass sich Ärzte auch um ihre eigene Gesundheit zu kümmern haben, um für den Patienten die beste Gesundheitsversorgung zu ermöglichen: «I WILL ATTEND TO my own health, well-being, and abilities in order to provide care of the highest standard.»

Zudem hat der Weltärztebund die einzelnen Paragraphen in eine neue Reihenfolge gebracht. Nach dem einleitenden Satz werden zunächst die Verpflichtungen gegenüber dem Patienten genannt, dann gegenüber der Profession und zuletzt gegenüber der Gesellschaft angeführt.

\section{Ökonomisierung}

2 https://www.wma.net/ policies-post/

wma-statement-on-physicians-well-being/.

Literatur zur Evidenz siehe bei Wiesing, Urban: Ethical Aspects of Limiting Residents' Work Hours. Bioethics 21 (2007), S. 398-405.

3 Rheinsberg, Zoe, Kloiber Otmar, Parsa-Parsi, Ramin, Wiesing, Urban: Medical oath: Use and relevance of the Declaration of Geneva. A survey of member organizations of the World Medical Association (WMA). Medicine, Health Care and Philosophy 2017, doi 10.1007/ s11019-017-9794-x
Im Weltärztebund wird in zahlreichen Zusammenhängen darüber diskutiert, dass die Medizin zwar ökonomisches Denken berücksichtigen muss, dieses aber in ein Spannungsverhältnis zur Medizin geraten kann. Und zwar in ganz unterschiedlichen Richtungen: Weltweit gesehen dürften überwiegend ökonomische Beschränkungen aufgrund von mangelnden Ressourcen die Medizin eingrenzen; in den wohlhabenden Ländern sieht man sich zudem mit Überversorgung und mit Übertherapie aufgrund von ökonomischen Anreizen konfrontiert. Ein Eid mit dem Anspruch auf Weltgültigkeit steht vor der Aufgabe, auf diese unterschiedlichen Phänomene mit einer möglichst kurzen Formulierung zu reagieren. In der Revision hat der Weltärztebund in dem Satz «I WILL PRACTISE my pro- fession with conscience and dignity" mit der Ergänzung "and in accordance with good medical practice» auf wissenschaftliche Standards hingewiesen, die ein Arzt in seiner Tätigkeit einhalten muss. Diese Erwähnung soll auch einer unangemessenen Ökonomisierung entgegenwirken. Unnötige Interventionen entsprechen eben gerade nicht der good medical practice, genau wie eine Unterversorgung.

\section{Das Genfer Gelöbnis und seine Verbreitung}

Die faktische Verbreitung und Nutzung des Gelöbnisses kann den selbstgesetzten Anspruch nicht erfüllen, das Gelöbnis aller Ärztinnen und Ärzte zu sein. Eine Untersuchung im Rahmen der Revision musste feststellen, dass das Genfer Gelöbnis in dieser Hinsicht weit hinter den Erwartungen zurückbleibt. ${ }^{3}$ Nicht zuletzt deshalb wurden die Eingangsworte "At the time of being admitted as a member of the medical profession" mit den ersten Worten "At the time of being admitted" gekürzt. Demnach ist der Eid auch bei anderen Gelegenheiten zu nutzen als nur bei der Aufnahme in die ärztliche Profession. Um den Anspruch noch klarer hervorzuheben und die Sichtbarkeit in den neuen Medien zu erhöhen, wurde der Untertitel «The Physician's Pledge» hinzugefügt. Das Genfer Gelöbnis wurde bisher nur schwer von Internet-Suchmaschinen erfasst. Die unzureichende Verbreitung des Gelöbnisses wurde auch auf der Generalversammlung des Weltärztebundes thematisiert, die sich darin einig war, dass weitere Massnahmen unternommen werden müssen, um dem Gelöbnis auch faktisch die Stellung zukommen zu lassen, die es beansprucht und verdient. Denn sie war überzeugt, dass es im Zeitalter der Globalisierung für die Profession unverzichtbar ist, weltweit einen gemeinsamen ethischen Standard zu besitzen. So sind Sektionen auf internationalen Konferenzen geplant, die das Problem einer gemeinsamen ethischen Grundlage der ärztlichen Profession im Zeitalter der Globalisierung ansprechen. Zudem will der Weltärztebund $a b$ sofort das Gelöbnis zu Beginn seiner jährlichen Generalversammlungen verlesen.

\section{Profession in einer globalisierten Welt}

Das Genfer Gelöbnis ist kein Selbstzweck. Es adressiert ein dringendes Problem der gegenwärtigen Medizin, und man sollte das Bemühen um weitere Verbreitung als eine Antwort auf eine Entwicklung verstehen, die nicht nur die Medizin beeinflusst: die Globalisierung. In einer solchen Welt stellt sich die Frage, was die Profession in ethischer Hinsicht zusammenhält und wie 
man Tendenzen einer Deprofessionalisierung entgegentreten kann. Gibt es einen Kern von moralischen Normen, den eine Ärztin oder ein Arzt in allen Bereichen dieser Welt zu beachten hat? Auf diese Fragen kann es nur eine globale Antwort geben. Nationale Eide können auf dieses Problem naturgemäss keine
Antwort geben. Der Weltärztebund hat mit der Verabschiedung ein Gelöbnis für 114 Ärztekammern bzw. -verbände verbindlich gemacht und so einen Kern von Normen für die allermeisten Ärzteverbände der Welt aufgestellt, der die wichtigsten und eben unverzichtbaren Grundlagen der ärztlichen Profession darstellt.

\section{WELTÄRZTEBUND}

\section{DEKLARATION VON GENF}

verabschiedet von der

2. Generalversammlung des Weltärztebundes, Genf, Schweiz, September 1948, und revidiert von der

22. Generalversammlung des Weltärztebundes, Sydney, Australien, August 1968, und revidiert von der

35. Generalversammlung des Weltärztebundes, Venedig, Italien, Oktober 1983, und revidiert von der 46. Generalversammlung des Weltärztebundes, Stockholm, Schweden, September 1994, und sprachlich überarbeitet auf der

170. Vorstandssitzung, Divonne-les-Bains, Frankreich, Mai 2005, und auf der 173. Vorstandssitzung, Divonne-les-Bains, Frankreich, Mai 2006, und revidiert von der

68. Generalversammlung des Weltärztebundes, Chicago, Vereinigte Staaten von Amerika, Oktober 2017

\section{Das ärztliche Gelöbnis}

\section{Als Mitglied der ärztlichen Profession}

gelobe ich feierlich, mein Leben in den Dienst der Menschlichkeit zu stellen.

Die Gesundheit und das Wohlergehen meiner Patientin oder meines Patienten werden mein oberstes Anliegen sein.

Ich werde die Autonomie und die Würde meiner Patientin oder meines Patienten respektieren. Ich werde den höchsten Respekt vor menschlichem Leben wahren.

Ich werde nicht zulassen, dass Erwägungen von Alter, Krankheit oder Behinderung, Glaube, ethnischer Herkunft, Geschlecht, Staatsangehörigkeit, politischer Zugehörigkeit, Rasse, sexueller Orientierung, sozialer Stellung oder jeglicher anderer Faktoren zwischen meine Pflichten und meine Patientin oder meinen Patienten treten.

Ich werde die mir anvertrauten Geheimnisse auch über den Tod der Patientin oder des Patienten hinaus wahren.

Ich werde meinen Beruf nach bestem Wissen und Gewissen, mit Würde und im Einklang mit guter medizinischer Praxis ausüben.

Ich werde die Ehre und die edlen Traditionen des ärztlichen Berufes fördern.

Ich werde meinen Lehrerinnen und Lehrern, meinen Kolleginnen und Kollegen und meinen Schülerinnen und Schülern die ihnen gebührende Achtung und Dankbarkeit erweisen.

Ich werde mein medizinisches Wissen zum Wohle der Patientin oder des Patienten und zur Verbesserung der Gesundheitsversorgung teilen.

Ich werde auf meine eigene Gesundheit, mein Wohlergehen und meine Fähigkeiten achten, um eine Behandlung auf höchstem Niveau leisten zu können. 